Meta

Journal des traducteurs

Translators' Journal

\title{
Le vocabulaire de l'athlétisme
}

\section{Donald E. Schlesinger}

Volume 20, numéro 4, décembre 1975

URI : https://id.erudit.org/iderudit/004042ar

DOI : https://doi.org/10.7202/004042ar

Aller au sommaire du numéro

Éditeur(s)

Les Presses de l'Université de Montréal

ISSN

0026-0452 (imprimé)

1492-1421 (numérique)

Découvrir la revue

Citer cet article

Schlesinger, D. E. (1975). Le vocabulaire de l'athlétisme. Meta, 20(4), 308-313.

https://doi.org/10.7202/004042ar d'utilisation que vous pouvez consulter en ligne.

https://apropos.erudit.org/fr/usagers/politique-dutilisation/ 


\section{DROBLEMES ET SOLUTIONS}

\section{LE VOCABULAIRE DE L'ATHLÉTISME}

Il y a trois ans, lors des Jeux olympiques (Olympic Games) de Munich, nous avons rédigé un petit article sur les «Pièges de la terminologie sportive » qui a paru dans le numéro de décembre 1972 de Traduire. Notre étude, nécessairement incomplète et subjective, ne prétendait en aucune façon être exhaustive, surtout lorsqu'on considère la grande variété de sports qu'elle a traitée. Trois ans plus tard, à la veille des Jeux de Montréal, nous reprenons notre plume, cette fois pour analyser le vocabulaire d'un seul sport, le plus ancien et le plus universellement pratiqué de tous, l'athlétisme ( track and field).

Commençons par des termes généraux qui s'appliquent à plus d'une discipline (event) avant de reprendre ensuite chaque course ou concours individuellement pour en dégager l'essentiel du vocabulaire qui s'y rapporte. Les différentes épreuves (events) se déroulent sur un stade (in a stadium) qui comporte les tribunes (stands ou grandstand), la piste (track), parfois en cendrée (cinder track) mais plus souvent, de nos jours, en tartan (du moins pour les grandes rencontres), et une pelouse (infield). Les meilleurs stades ont un tableau d'affichage (scoreboard) qui tient le spectateur au courant des performances ainsi que du score du match s'il s'agit d'une rencontre (meet) par équipes.

Il est bien entendu que les courses (races, track ou running events) se disputent sur la piste, alors que c'est sur la pelouse qu'ont lieu les concours (field events). La piste elle-même est divisée en couloirs (lanes) et mesure 400 mètres (ou 440 yards) le tour. Elle comporte deux lignes droites (straightaways) dont une s'appelle la ligne opposée (backstretch) et l'autre, faute de mieux, la ligne droite (homestretch), ainsi que deux virages (turns).

Avant de courir, de sauter ou de lancer, chaque concurrent (competitor) exécute rituellement des exercices d'assouplissement (stretching and bending exercises), car s'il ne s'échauffe pas (warm up) comme il faut, l'athlète risque de se blesser en claquant un muscle (pulling a muscle) lors de la compétition. Les claquages (muscle pulls ou pulls tout court) étant le fléau des athlètes, on comprend facilement l'importance accordée à ces exercices préliminaires. Quand il se sent bien à l'aise, le concurrent, toujours en survêtement (sweatsuit), se repose 
pour attendre le début de son épreuve. Nous allons suivre, avec lui, le déroulement d'un match imaginaire.

Les courses se divisent en cinq catégories : courses de vitesse, ou sprints (en français comme en anglais), courses de demi-fond (middle distance races), courses de fond (distance races), courses d'obstacles (hurdle races) et courses de relais (relay races). La plus courte de toutes, celle aux Jeux qui donnent au gagnant le droit de s'appeler «L'être le plus rapide du monde » («The World's Fastest Human $\gg$ ), est le 100 mètres. Nous voilà sur la ligne du départ (starting line). Le starter crie « $\mathbf{A}$ vos marques» ( On your marks, « Take your marks, 》 ou «Go to your marks») et les huit finalistes se mettent lentement sur «quatre pattes \& pour prendre leur départ dit accroupi (crouch start). Chacun se sert d'un starting-block pour caler ses pieds, et l'on s'aperçoit que certains des sprinters préfèrent mettre les cales (blocks) très près de la ligne en prenant un départ groupé (bunch start ou, moins souvent, bunched start)alors que d'autres, en général plus grands de taille, prennent un départ allongé (elongated start) en éloignant les cales de la ligne. À la deuxième commande, «Prêts» ( Set »), tous se relèvent partiellement en se basculant vers l'avant et restent complètement immobiles à attendre le coup de feu (gun) du starter. Celui qui, tout en restant derrière la ligne, est en mouvement au moment du feu est dit avoir exécuté un départ en baseule (rolling start), ce qui est illégal. De même, celui qui part avant le feu exécute un faux départ (false start ou break).

L'épreuve s'engage et, comme c'est souvent le cas, l'arrivée d'une course de sprint peut être extrêmement serrée (tight). Il est bien probable que plusieurs sprinters se trouvent sur une même ligne : une véritable arrivée dans un mouchoir (blanket finish) ! Celui qui a le meilleur cassé (lean) sera déclaré vainqueur, mais il faudra la photo-finish pour vérifier le résultat. Le terme est intéressant car «photo-finish » a deux sens en français : il désigne à la fois l'enregistrement photographique de l'arrivée d'une course et l'appareil qui l'effectue. Or, on dira en anglais "photo» tout court pour le premier sens ( timer 》 (car l'appareil enregistre le «chrono » en même temps) pour le second. La "photo-finish» en anglais correspond uniquement à l'arrivée elle-même. Les chronométreurs (timers) consultent leurs chronomètres (stopwatches), car tout le monde s'intéresse au temps ou « chrono » (time) du gagnant. Il est vrai que de nos jours, surtout au niveau de la haute compétition, le chronométrage électrique (automatic ou electric timing) est de rigueur.

Souvent, le vainqueur du 100 mètres tentera un doublé (double) en s'inscrivant (entering) au 200 mètres. D'autres, fatigués par les séries (trial heats), quarts de finale (quarter finals), et demi-finales (semi-finals) du 100 , déclareront forfait (scratch) et ne disputent que la plus courte des deux courses. À la différence du 100 mètres où tous les concurrents prennent leur départ sur une même ligne, le départ du 200 se donne en décalage (staggered start). À l'entrée de la courbe (turn ou curve), celui qui se trouve à la corde (on the rail, pole, ou inside) semble être handicapé par rapport aux autres. Tout s'égalise, cependant, à la sortie du virage (off the turn) où, théoriquement, les meilleurs sprinters se retrouvent sur la même ligne. 
Le 400 mètres est non seulement le plus long des sprints mais aussi le plus éreintant. Il ne suffit pas d'être le plus rapide, car ici intervient la notion de répartition de l'effort. La résistance (stamina) entre en jeu et l'on doit apprendre le sprint contrôlé (float) pour bien réussir sur cette distance exigeante. Celui qui part trop rapidement risque fort de se crisper (tighten up, tie up, get tight, ou, dans la langue familière, "catch rig, » de rigor mortis !) à l'entrée de la dernière ligne droite. Les 100 derniers mètres deviennent alors un véritable cauchemar : on lit la douleur sur le visage de l'athlète, sa foulée (stride) se raccourcit. Bref, la crispation (tying up, tightening up) est l'ennemi du coureur du 400 mètres.

Quittons le monde des sprinters pour entrer dans le royaume des coureurs de demi-fond (middle distance runners). Deux courses dominent: le 800 mètres ( 880 yards ou half mile) et la plus prestigieuse de toutes les courses, le mile (ou son équivalent métrique, le 1500 mètres). Adieu starting-blocks et départ accroupi. On prend un départ debout (standing start) pour ces deux courses ainsi que pour toutes les courses d'une distance supérieure à 400 mètres. À l'encontre des sprints aussi, la tactique joue un rôle capital dans le demi-fond, car l'idée d'allure ou de train (pace) prime tout. Va-t-on donner l'allure ? Si l'on mène le train (sets the pace) on risque de se faire coiffer sur la ligne (to be caught from behind, to be nipped at the wire) par un bon finisseur (kicker) au coup de reins (finishing kick, ou kick tout court) dévastateur. Par contre, si l'on reste dans le peloton (pack), on risque non seulement les à-coups (elbowing, jostling) ou plus sérieusement encore, les coups de pointe (spiking) de ses adversaires, mais aussi la possibilité d'être enfermé (boxed in) par deux ou trois coureurs placés directement devant soi et à droite.

A chaque tour de piste (lap), on annonce les temps de passage (splits, du verbe «to split, » employé dans le sens de « diviser ») du meneur. Ces temps intermédiaires sont très importants, car ils permettent au coureur comme au spectateur de prévoir plus ou moins le temps final que réussira l'athlète. En Europe, on signale le dernier tour d'une course de fond ou de demi-fond en sonnant une cloche. En Amérique, ce tour de cloche (bell lap) est remplacé, le plus souvent, par un « gun lap, » car on préfère, quand il ne reste plus qu'un tour à la course, tirer un coup de revolver dans l'air.

Les trois grandes courses de fond $(5000 \mathrm{~m}$, ou bien 3 miles; $10000 \mathrm{~m}$ ou 6 miles ; et le marathon, c'est-à-dire 26 miles 385 yards !) offrent peu de termes de vocabulaire au traducteur. Toutefois, mentionnons-en au moins deux, le second souffle (second wind) qui se rapporte au taux de consommation de l'oxygène (oxygen consumption rate) du coureur, et le bel exploit de faire cavalier seul (to be all alone, way out in front, run by oneself) qui n'arrive en général que dans les courses les plus longues.

Passons des courses plates (flat races) aux courses d'obstacles (hurdle races) qui comptent le $110 \mathrm{~m}$ haies (120 yard high hurdles), le $\mathbf{4 0 0} \mathrm{m}$ haies (440 yard intermediate hurdles) et le steeple (Steeplechase). Ici, c'est surtout la technique qui décide l'ordre de l'arrivée et qui sépare le gagnant des autres. Le passage (clearing) de la haie doit s'effectuer, bien entendu, dans la foulé (in full stride) et bien que l'on ne soit pas pénalisé pour avoir heurté un obstacle (hit a hurdle), on 
perd plusieurs dixièmes de seconde en ce faisant. De même, on perd du temps si, en trouvant que sa jambe d'attaque (lead leg) est trop loin ou trop près de la haie, on piétine devant l'obstacle (chops one's steps). Finalement, on risque de se faire déclasser (disqualified) si, en franchissant (clearing) la haie, on passe sa jambe d'appui (trailing leg) aUtour de l'obstacle.

A toute la terminologie des courses de haies s'ajoute un seul terme, très joli d'ailleurs, qui s'applique au 3000 mètres steeple. Il s'agit de la rivière (water-jump) dont le passage, très amusant pour la foule, l'est beaucoup moins pour le coureur !

Les courses de relais, surtout le $4 \times 100$ mètres (440 yard relay), sont parfois très spectaculaires et nous fournissent, en même temps, un vocabulaire assez spécialisé. Il s'agit, dans le $4 \times 100 \mathrm{~m}$, d'un relais de quatre coureurs effectuant chacun son parcours (leg). Le dernier parcours (anchor leg) est assuré, en général, par le meilleur des quatre relayeurs (anchor $\mathrm{man}$ ). Les deuxième, troisième et quatrième coureurs se placent en position d'attente au début de leur zone de transmission (passing zone) et attendent, chacun à son tour, que le relayé (incoming man) soit à une distance d'environ six mètres avant de démarrer à toute vitesse quand leur coéquipier passe (hits) au niveau d'un repère (mark) préalablement placé sur la piste. Les relayeurs se transmettent (pass) un cylindre de bois ou de métal qui s'appelle le témoin (baton). Il va sans dire que les transmissions ou prises du témoin (baton passes, stick passes ou exchanges) exigent de la part du relayé et du relayeur (outgoing man) une mise au point et une synchronisation des vitesses très précises. Il est logique que nous terminions notre étude des courses par le $\mathbf{4} \times \mathbf{4 0 0}$ mètres (mile relay) car, traditionnellement, c'est ce relais qui clôture une rencontre d'athlétisme.

Mais les courses n'expliquent que la première partie de l'expression \& track and FIELD ». En effet, les concours (field events) nous attendent : sur la pelouse, tout se passe en même temps. C'est un véritable cirque et l'on ne sait pas où donner de la tête. À gauche, c'est le saut en longueur (long jump, autrefois, broad jump) qui attire notre attention. Voilà le premier sauteur qui, après avoir étalonné (measured) très soigneusement ses appuis (steps) sur la piste d'élan (runway), se retourne pour prendre son élan (approach). La vitesse de sa course d'élan augmente à mesure qu'il s'approche de la planche d'appel (take-off board). Il pose son pied d'appel (take-off foot) sur la planche et s'envole vers la fosse de réception (landing pit). Une fois dans l'air, il exécutera un ciseau des jambes (hitchkick) et parfois même un double ciseau (double hitchkick) non point pour augmenter sa distance finale, ce qui n'est plus possible, mais simplement pour mieux maintenir l'équilibre de son corps. Le drapeau rouge nous indique que le sauteur a dépassé la planche d'appel et a donc « mordu» (fouled).

De l'autre côté de la piste, le saut en hauteur (high jump) se dispute. Les sauteurs s'échauffent sur le sautoir (jumping area) en attendant de prendre leur premier essai (attempt). Une barre (crossbar, ou bar tout court) est fixée sur les appuis (supports) de deux poteaux (standards) placés devant un matelas pneumatique ( air pit $»$ ) qui sert d'aire de réception (landing area). Plusieurs concurrents ont abandonné la technique classique du rouleau ventral (straddle) ou californien (western roll) pour pratiquer le « fosbury flop » à la manière de Dick Fosbury qui, 
le premier, a tourné le dos à la barre juste avant d'amorcer le saut et a donc inventé le style «dorsal. » Le sauteur plus conventionnel prend son élan, pose sa jambe d'impulsion (take-off leg) devant la barre, lance la jambe libre (lead leg) vers la barre, puis essaie de franchir (clear) la barre en enroulant son corps et finalement sa jambe d'appel (devenu en anglais «trailing leg, » une fois dans l'air). $\mathrm{Si}$, à la fin de la compétition, deux sauteurs restent ex requo (tied), on départage les athlètes « aux essais » (on fewer misses), le gagnant étant celui qui a franchi la dernière hauteur au plus petit nombre d'essais.

Alors que le triple saut (triple jump, autrefois hop, step and jump) ne nous offre pas d'autres termes techniques, on ne peut pas en dire de même pour le saut à la perche (pole vault). Les perchistes (pole vaulters) sont, en quelque sorte, les acrobates de l'athlétisme car, à la vérité, le sauteur à la perche est à la fois un coureur à pied, un sauteur et un gymnaste. Le voilà qui, portant sa perche en fibre de verre (fiberglass pole), se lance vers le point de piqué (plant) où il place l'extrémité de sa perche dans le butoir ou bac (box) avant d'amorcer le saut. Le spectateur retient son souffle pendant que le perchiste s'envole vers le ciel (ou presque !).

Moins spectaculaires que le saut à la perche, les quatre lancers (throws) méritent néanmoins qu'on s'y arrête. Tout au milieu de la pelouse, on lance le javelot (javelin), mais pour des raisons évidentes, on préfère un endroit éloigné pour disputer le lancer du marteau (hammer throw). Le lanceur est entouré d'une cage, car parfois le fil (chain) attachant la poignée (handle) au marteau se casse et la force centrifuge (centrifugal force) lance le marteau indépendamment de la volonté du lanceur! Mais les accidents sont rares, et normalement, on regarde le lanceur effectuer d'abord quelques moulinets (winds ou, moins souvent, swings) et ensuite plusieurs tours (turns) avant de lancer.

Le lancement du poids (shot put) est moins spectaculaire que les autres lancers. Il s'agit d'un engin (implement) rond pesant 7257 kilos (16 pounds) que l'on essaie de lancer le plus loin possible. Pour ce faire, on se met à l'intérieur d'une aire de lancer en forme de cercle (the circle). Dos à la direction du jet, on traverse d'un sursaut le cercle en prenant soin de ne pas dépasser le butoir (toeboard ou stopboard qui se trouve à l'avant du cercle.

En dernier lieu, mentionnons le lancer du disque (discus throw), spécialité pratiquée lors des Jeux antiques d'Olympie. Mentionnons aussi, pour mémoire, la marche (walk) et le décathlon (decathlon) qui, malheureusement, ne fournissent pas d'autres termes spéciaux.

Nous terminons cette étude de la terminologie de l'athlétisme en considérant les vêtements très simples que porte l'athlète. En plus du survêtement, le coureur porte évidemment un maillot (jersey ou top) et une culotte (shorts ou pants). Sous la culotte, il porte un suspensoir (athletic supporter, ou plus familièrement, 《jockstrap » ou «jock») et, aux pieds, des chaussures à pointes (spiked shoes) ou des pointes (spikes) tout court. Lors d'une rencontre, il portera un dossard (number) numéroté.

Nous espérons que cet aperçu sur le vocabulaire de l'athlétisme profitera non seulement aux traducteurs du domaine sportif mais aussi aux lecteurs- $\ll$ fanas $»$ de 
sports de Meta qui se demandent parfois « comment on dit cela » en français ou en anglais. À tous ceux qui attendent, avec nous, la cérémonie d'ouverture des Jeux de Montréal, nous vous rappelons la devise de l'Olympisme: "Citius, Altius, Fortius. » Que les Jeux commencent! (Let the Games begin!).

Donald E. SChLESINGER 